\title{
Traduire
}

Ine autre perspective sur la tataduction

Revue française de la traduction

$234 \mid 2016$

La traduction, un sport de haut niveau

\section{Figurines : portraits en prose et poésie de joueurs de football}

Bernard Vanel, Sylvain Guichard, Roberto Veracini, Fabrizio Parrini

\section{Vanessa De Pizzol}

\section{(2) OpenEdition}

\section{Journals}

Édition électronique

URL : http://journals.openedition.org/traduire/817

DOI : $10.4000 /$ traduire.817

ISSN : 2272-9992

Éditeur

Société française des traducteurs

Édition imprimée

Date de publication : 15 juin 2016

Pagination : 87-91

ISSN : 0395-773X

Référence électronique

Vanessa De Pizzol, «Figurines : portraits en prose et poésie de joueurs de football », Traduire [En ligne], 234 | 2016, mis en ligne le 07 décembre 2016, consulté le 25 septembre 2020. URL : http:// journals.openedition.org/traduire/817 ; DOI : https://doi.org/10.4000/traduire.817 


\section{Compte rendu d'ouvrage \\ Figurines : portraits en prose \\ et poésie de joueurs de football \\ Bernard Vanel, Sylvain Guichard, \\ Roberto Veracini, Fabrizio Parrini}

Vanessa De Pizzol

\section{Quand le rectangle vert se fait poésie}

"Figurines ", un mot qui fait mouche aussitôt dans l'univers du ballon rond, car il nous ramène immanquablement aux figurines Panini qui traversent les décennies depuis 1960 et réunissent toutes les générations autour d'une même passion.

Ce petit ouvrage s'inscrit précisément dans cette tradition, l'objectif commercial en moins. L'amour inconditionnel pour le foot y occupe la première place et les idoles qui font palpiter le cœur des supporters sont passées en revue. Quatre auteurs partagent leur " collection " de figurines avec le lecteur, pour son plus grand plaisir. Chacun d'entre eux nous présente son équipe idéale, à travers une série de onze portraits, en poésie ou en prose. Sous les regards croisés de deux auteurs français et deux auteurs italiens, c'est tout un pan de l'histoire du football et des destins sublimes ou tragiques de ces joueurs qui ressurgit.

Comme dans un album de photos de famille, les auteurs se replongent avec délectation dans leur enfance, leur propre passé, et, avec parfois un brin de nostalgie, revivent les moments forts de ce sport qui ont marqué leurs vies comme des nations entières.

Individuelles et collectives, les destinées s'entremêlent de façon inextricable et les noms de prestigieux athlètes côtoient d'autres noms que les années ont fait sombrer dans l'oubli. La passion du ballon est un fil d'Ariane qui relie les conversations enflammées des supporters dans les bars, les discussions animées au sein des familles, les retransmissions à la radio, les commentaires des journalistes sportifs, les matchs à la télévision...

Cet objet littéraire insolite nous rappelle que le football est bien plus qu'un sport. Jean Djorkaeff, dans la préface de ce recueil, va même jusqu'à affirmer qu'il peut s'apparenter à un extraordinaire ciment social et faire la preuve de l'union admirable du corps et de l'esprit (" faire vivre un ballon avec ses pieds et sa tête(1) "). Quant au poète toscan Alessandro Agostinelli,

(1) Bernard Vanel, Sylvain Guichard, Roberto Veracini, Fabrizio Parrini, Figurines. Portraits en prose et poésie de joueurs de football, Vénissieux, Éditions La passe du vent, mars 2016, p. 5. 
qui signe l'introduction, le football reste pour lui avant tout un vecteur d'émotions fortes (" [... ] une des plus grandes émotions de ma vie, ce fut précisément lorsque je p[us] participer à ma première réunion du club de notre équipe de cœur et prendre ma carte d'adhérent(2) "), voire un rite initiatique (" Le football était le sujet incontournable de ces discussions de grands et j'avais le privilège de pouvoir être là au milieu [...](3) "). Dépassant le strict cadre de la discipline sportive, puisqu'il est question du "style(4) " des joueurs, qui, pour certains, sont comparés à des "danseurs sur le rectangle de jeu ", cet art du ballon marque incontestablement la vie de ceux qui le pratiquent et l'adulent.

La galerie de portraits de Fabrizio Parrini, poète nourri par le théâtre et l'enseignement de l'histoire de l'art, se présente comme une succession de " figures épiques qui montrent le football comme une des expressions les plus fascinantes de la culture des hommes(5) ". II est vrai que de nombreux intellectuels s'insèrent dans cette tradition, d'Albert Camus, gardien de but dans une équipe en Algérie, à Pier Paolo Pasolini, qui pratiquait le foot sur les terrains vagues de la banlieue romaine, dans sa variante "populaire ", au sens noble du terme.

Les portraits de footballeurs chers à Parrini, déclinés en onze poèmes, sont véritablement frappés au sceau du destin. Le plus emblématique, à ce titre, reste sans aucun doute celui d'Obdulio Varela (1917-1996). Le poète choisit de retracer, de l'intérieur, le moment où l'existence de ce natif de Montevideo bascule, au Maracana. Coupe du monde de 1950 : l'équipe uruguayenne dont il est le capitaine affronte le Brésil à domicile. Les dés semblent jetés : " [...] nous étions morts mais comment pouvions-nous / nous rendre devant ceux qui soutenaient / être l'équipe de Dieu(6) „. Puis vient l'heure du grand chamboulement : "Quand Schiaffino a marqué / le stade a eu comme un frisson / qui est devenu un murmure de peur. / Moi je l'ai vue dans les yeux des Brésiliens / qui cherchaient des explications. / [...] Puis Ghiggia a inventé un tir / tordu qui a exécuté Barbosa / le gardien noir du Vasco / et toutes ces deux cent mille / personnes que j'ai vues en larmes / dans le silence d'une défaite inattendue. / Je jure que je ne savais pas quoi faire. / Nous nous sommes embrassés au milieu / de tout un peuple en larmes(7) ". C'est la nuit, il quitte son hôtel et la fête pour aller arpenter Copacabana et finit par entrer dans un bar, où on le reconnaît : "Un des clients vient vers moi / et m'embrasse tu es un grand capitaine Obdulio -/ me dit-il - Puis il pleure et ne sait pas quoi dire [...]". Pour ce capitaine resté dans l'histoire, la victoire a un goût amer, il a le sentiment d'avoir " profané / la cathédrale du football(8)".
(2) Ibid., p. 6.
(3) Ibid., p. 7.
(4) Ibidem.
(5) Ibid., p. 10.
(6) Ibid., p. 33.
(7) Ibidem.
(8) Ibid., p. 34. 
Nombreuses sont les trajectoires sportives et humaines, dans la lumière ou dans l'ombre, qui s'entrecroisent au gré des pages. Comme celle de Nacka Skoglund, footballeur suédois, qui finit misérablement son existence dans les foires et " fait le phoque pour faire rire / les enfants jusqu'à ce qu'il se jette la tête la première / dans la mort au numéro 42 / de Katarina Bangata juste à proximité / de l'endroit où Greta Garbo / était devenue une étoile(9)".

Bernard Vanel, né en 1951 et supporter passionné de l'équipe de Nantes, propose à travers sa collection de "Rebonds " une image nuancée de l'univers du football où les héros du terrain, malgré leurs exploits, restent à taille humaine. Parmi eux, André Kabile, qui, dans ses années de gloire " [...] était un roc. Une tour imprenable sur l'échiquier des stades(10) ", une marque de fabrique qu'il a longtemps gardée : "[i]l n'était plus professionnel et [...] [à] quarantedeux ans - la petite moustache, les chaussettes baissées -, il n'en restait pas moins encore un monument(11) ॥. Les physiques improbables y trouvent également leur place :

Qui se souvient encore de Jean-Claude Baulu ? [...] [D]e son mètre soixante-cinq, [il] vint délivrer les Verts sous l'œil du Général.

Ce fut l'heure de gloire de ce petit ailier au crâne dégarni qu'on aurait sans ce but oublié sûrement dans des archives poussiéreuses(12).

Vanel se plaît encore à brosser le portrait d'un footballeur brillant et atypique, épris de culture, diplômé en droit, et profondément engagé. Eugène N'Jo Lea (1931-2006) est reconnaissable sur le rectangle vert " par sa classe, ses ailes de pigeon, sa souplesse de liane, sa puissance et ses dribbles. II assume son nom qui veut dire "panthère" en dialecte africain(13) „. Mais il abandonne cet univers par la suite : "II devint diplomate. [...] C'était par accident qu'il avait été footballeur. II le disait souvent. Mais il avait compris que le football, un jour, pourrait participer, peut-être, à la promotion sociale de frères africains. II rêvait de donner les moyens à l'Afrique de gagner la Coupe du monde. On ne l'entendit pas. Même on le menaça. Eugène fut déçu. Puis il tomba malade(14)".

Le " petit dictionnaire poétique du football " de Roberto Veracini propose onze définitions de l'arte del calcio. Des instantanés de talentueux joueurs se succèdent les uns aux autres, comme saisis par l'œil nostalgique d'un admirateur qui révèle, par endroits, l'absurdité de leur condition de footballeur. Ces champions du ballon semblent toujours quelque peu décalés sur le terrain. Giacinto Facchetti, figure de l'ailier gauche, est qualifié de " sprinteur pur et infatigable, élégant et invincible. Un athlète tout terrain, arrivé qui sait comment dans le monde du foot-
(9) Ibid., p. 57.
(10) Ibid., p. 61.
(11) Ibid., p. 61.
(12) Ibid., p. 68.
(13) Ibid., p. 73.
(14) Ibidem. 
ball, un géant débonnaire qui réécrivait l'histoire du football moderne(15) ». Le milieu offensif Giancarlo Pasinato demeure dans les mémoires " pour ses courses puissantes, solitaires et impossibles, qui rappelaient les antiques cavaliers invincibles, mais très fragiles cependant, dans leur poursuite désespérée de rêves et de ballons lointains(16) ". Mariolino Corso, quant à lui, incarne un ailier gauche bien singulier, tant par sa technique de jeu que par son allure. Étrangement, il se déplace très peu sur la pelouse : " [il] jouait sa partie dans un mètre carré de terrain et ne courait pas. De cet avant-poste, il scrutait l'horizon, en cherchant dans l'air une ouverture étroite, la fissure imperceptible dans le placement adverse(17) ". Élément plus inattendu encore, son apparence physique est en soi une antithèse du corps athlétique qu'un sportif de ce niveau devrait arborer : " Mariolino Corso avait aussi le bedon, qui, chez un footballeur, est signe sans équivoque de classe supérieure(18) „. Mais c'est incontestablement la figurine $n^{\circ} 10$, celle d'Evaristo Beccalossi, dénommé " fantaisiste ", qui illustre le mieux le football selon Roberto Veracini : " II jouait comme un garçon distrait, que l'on avait mis là presque par hasard, un peu renfrogné, vaguement mélancolique... puis pourtant arrivait le ballon et le garçon avait un sursaut, il se transformait, commençait à inventer, il projetait ses rêves dans cet objet sphérique, qui devenait imprenable [...](19) ॥. Être là sans y être, telle serait donc la véritable philosophie du football...

Sylvain Guichard, acteur et metteur en scène, fait partie de la génération Zidane et tient à partager " les grandes émotions et l'attachement [qu'il] voue au sport collectif le plus populaire du monde, sans oublier sa face sombre et ses excès(20) ". C'est avec ses yeux d'enfant et son expérience de jeune joueur de football qu'il s'ouvre à cet univers. Parmi ses modèles, le gardien danois dont la stature tient de l'herculéen : "Schmeichel était un géant blond aux sourcils proéminents qui, une fois allongé dans les airs, les yeux rivés sur le ballon qui tente vainement de le fuir, représentait un tiers d'une cage de football(21) ". Quant à ses idoles, on compte Gabriel Batistuta, avant-centre argentin surnommé Batigol, un buteur extraordinaire, et bien entendu Éric Cantona et Zinédine Zidane.

C'est avec une joie sans mélange que Guichard revisite ses premières émotions de supporter, lesquelles restent intimement liées au temps de l'enfance. II recourt à l'anaphore pour tenter d'expliquer ce que cet état si particulier veut dire :

\footnotetext{
(15) Ibid., p. 81.

(16) Ibid., p. 83.

(17) Ibid., p. 97.

(18) Ibidem.

(19) Ibid., p. 95.

(20) Ibid., p. 98.

(21) Ibid., p. 100.
} 
Être supporter, c'est avoir la boule au ventre toute la journée précédant le match [...].

Etre supporter, c'est pouvoir passer la durée du match les yeux rivés sur le terrain, en se rongeant les ongles, contractant tous les muscles de son corps quand l'adversaire menace, et se sentir des ressorts dans les jambes quand les héros se rebiffent.

[...]

Être supporter, c'est mourir de déception ou exploser d'une joie démesurée, c'est se mettre dans des états absurdes, et tout ça n'a aucun sens commun, mais c'est délicieux. C'est incomparable(22).

Guichard ne triche pas, ne magnifie pas, il restitue simplement le regard émerveillé de l'enfant, de l'adolescent, face à la magie du ballon. II dessine sa géographie rêvée, établie en fonction de ses clubs préférés : " En Espagne, je ne jure que par l'Athletic Bilbao. En Angleterre, je vibre pour Leeds et Manchester United. Au Portugal, le Sporting. En Argentine, c'est River Plate. En Major League Soccer, je roule pour Montréal. En Suisse, pour les Young Boys de Berne. En Écosse, Heart of Midlothian. En Belgique, Club de Bruges. Aux Pays-Bas, I'Ajax Amsterdam. Et en Italie, la Fiorentina(23)! " Ce qui ne l'empêche pas d'exprimer des regrets à propos de la dérive financière qui gangrène le milieu du football : "Corruption des bas-fonds aux hautes sphères, agents véreux et paternalistes, contrats publicitaires, déficits abyssaux, milliardaires, investisseurs lunatiques, joueurs mercenaires. Je le sais, j'ai mal à mon sport fétiche. Et pourtant, je regarde toujours le foot(24)".

Le football n'est malheureusement pas le seul sport concerné par ces excès, mais les " Figurines " viennent confirmer que, génération après génération, la poésie du rectangle vert demeure bien la plus forte.

vanadep@orange.fr

\section{Figurines}

Portraits en prose et poésie de joueurs de football

VANEL Bernard, GUICHARD Sylvain, VERACINI Roberto, PARRINI Fabrizio

Préface de Jean Djorkaeff

Introduction d'Alessandro Agostinelli

Traduction de l'italien par Bernard Vanel

130 p. / Bilingue français-italien

Éditions La passe du vent, mars 2016

ISBN : 978-2-84562-282-1

Gencod : 3019000119305

(22) Ibid., p. 116.

(23) Ibid., p. 118.

(24) Ibid., p. 113. 